\section{Comment peut-on vivre avec des loups sauvages?}

\section{F. Burnier}

se dépeuplent depuis des décennies. C'est ainsi que, il y a quelques années, la commune voisine de Rochetta a Volturno a demandé à être intégrée au Parc, pour profiter elle aussi de ses retombées économiques. Une autre commune adjacente, Ortona nei Marsi, est actuellement en tractations dans le même sens;

- la situation n'a cependant de loin pas toujours été aussi favorable. Comme partout ailleurs, la haine viscérale du loup régnait, et avec elle les croyances les plus fantaisistes, tout particulièrement chez les villageois confinés dans les zones habitées. Ceux qui vivaient réellement dans le terrain, comme par exemple les bergers, avaient une attitude plus nuancée, comme on le verra plus loin. L'un des éléments décisifs dans la "réconciliation" de l'homme et du loup a été l'enclos (Area faunistica) de Civitella Alfedena, réalisé en 1972, dans lequel une meute de loups vivait en semi-liberté sous les yeux des habitants et des touristes. C'est ainsi que, la première année déjà, 120000 personnes vinrent visiter un village qui jusque là se mourait lentement.

\section{Moments inoubliables}

En cette aube de Pâques, après bien des affûts sans succès, nous eûmes l'occasion - tout à fait exceptionnelle d'après les gardes - d'observer pendant près d'une heure un ours et quatre loups, et d'étudier leurs comportements réciproques. La scène se termina lorsque l'ours se leva et s'en alla ostensiblement, ce qui permit enfin aux loups - la hiérarchie est évidente - de s'emparer des restes de la proie et de les traîner à l'abri des regards indiscrets. Pendant toute cette scène, trois biches paissaient tranquillement à quelques centaines de mètres de là.

Et aussi cette nuit étoilée de février où, du village enneigé, nous entendions, venant de la vaste pinède dominant la rivière, le chant d'un loup solitaire (je préfère le terme chant à celui de hurlement, à mon sens tout à fait déplacé; les Italiens disent ululato).

Ou encore cette "rencontre» nocturne avec un ours invisible qui nous avait repérés alors que nous étions à l'affût dans des fourrés, et qui, grognant et humant l'air, s'est approché à quelques mètres pour pouvoir nous identifier.

Enfin cette nuit passée sur un petit col enneigé, dans l'espoir d'une rencontre. Sous le ciel que les étoiles se partageaient avec les nuages, nous entendions rouler des pierres retournées par des sangliers à la recherche de quelque pitance, pendant que la chouette hulotte égrenait son chant. Vers le milieu de la nuit, les chiens de la bergerie se sont mis à aboyer en chœur; sans doute flairaient-ils la présence d'un loup à la rôde. Nous avons guetté longtemps et nous avons fini par nous rendormir alors qu'ils aboyaient toujours. Nous n'avons rien vu; mais, le matin, après avoir roulé nos sacs de couchage givrés, nous avons croisé une piste de loup toute fraîche dans la neige. 


\section{Figure 1}

Le village soigné de Civitella Alfedena doit toute sa prospérité à la présence du loup et de l'ours dans la région.

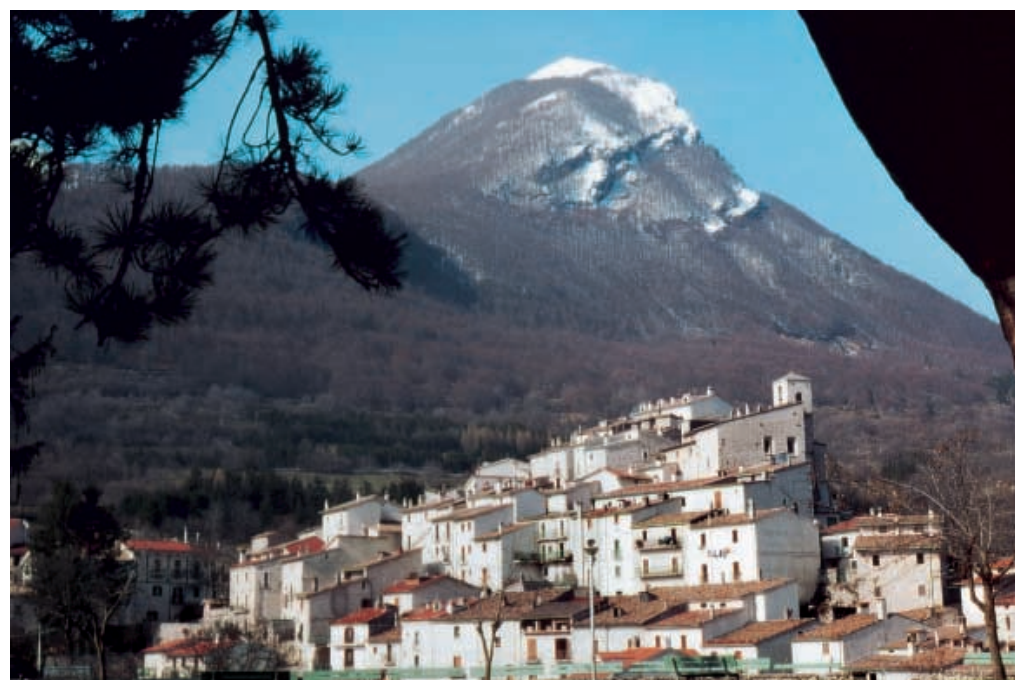

\section{Figure 2}

Une bergerie au pays des loups.

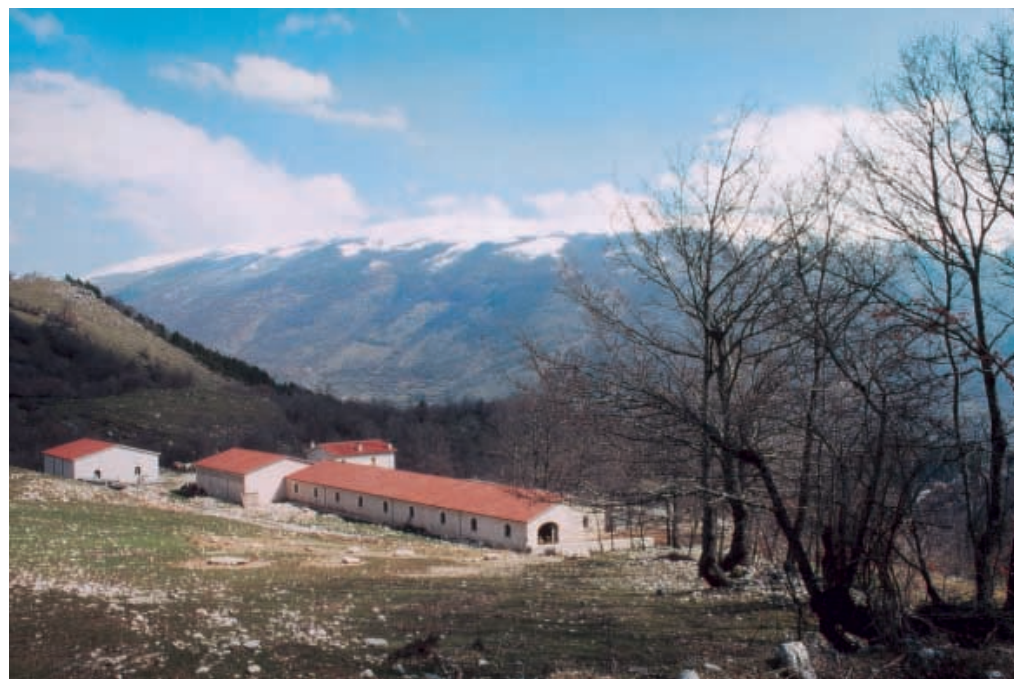

Où le loup vit-il?

"Promenons-nous dans les bois, pendant que le loup $n$ 'y est pas ....: en effet, bien souvent, c'est ailleurs qu'il se balade. Avec le renard et l'homme, le loup fait partie des espèces de mammifères les plus adaptables. La présence de nourriture en suffisance est pour lui plus importante qu'un type de biotope particulier. Steppes russes, plaines cultivées d'Espagne, forêts de Scandinavie, toundra gelée du Canada, abords des villes en Roumanie, nombreuses sont les adresses de notre grand carnivore sauvage. Les traces que nous avons observées à proximité immédiate des villages dans les Abruzzes n'ont donc rien d'exceptionnel, même en été, et sa présence est régulièrement constatée à Tivoli, soit a seulement vingt-trois kilomètres du Colisée. Une de ses principales qualités est sa discrétion: sitôt qu'ils restaient immobiles, ceux que nous avons observés se fondaient dans le paysage grâce aux couleurs de leurs pelages; par ailleurs, sa ressemblance avec le chien berger allemand permet au loup de passer incognito là où on s'attend plutôt à voir son cousin domestique. Le spécialiste italien Luigi Boitani, de l'Université de Rome, nous citait ce loup traversant la place de pique-nique du village d'Opi, dans le Parc National des Abruzzes, un aprèsmidi de ferragosto, sans attirer la moindre attention de la part des centaines de personnes venues s'y délasser sous un soleil torride.

Même pour la mise bas, le loup peut s'accommoder de la civilisation. Une portée de jeunes a été élevée avec succès dans une tanière située à quelques dizaines de mètres seulement d'une route fréquentée du Parc, à l'insu de ses nombreux utilisateurs.

\section{Le loup est-il dangereux?}

Cette question, nous l'avons posée partout où nous sommes passés.

- Au directeur du Parc et aux gardes tout d'abord: non, il n'existe aucun cas connu d'attaque contre des humains;

- à Nestore Campana, un berger de 89 ans ayant fait une cinquantaine de fois la transhumance depuis la côte adriatique: le loup et l'ours fuient toujours l'homme;

- à la patronne d'un restaurant, à la boulangère, au garagiste, au bûcheron: non, le loup s'enfuit dès qu'il s'aperçoit de la présence de l'homme: pas question d'en avoir peur: "niente paura!";

- enfin, et surtout, aux enfants des villages. Nous en avons interrogé beaucoup: ainsi ces quarantecinq enfants de l'école primaire de Villetta Barrea, rassemblés un matin pour répondre à nos questions. Sept ou huit d'entre eux avaient déjà eu l'occasion de rencontrer le loup en liberté. Leurs histoires commençaient de différentes manières mais elles finissaient toutes par: «... et quand il nous a vu, il s'est enfui!». Et ces trois filles d'une douzaine d'années, en vêtements de ski de fond, rencontrées dans le village citadelle d'Opi, et qui nous parlèrent de Biagio Di Santo, un jeune du village qui venait de gagner une médaille aux Jeux Olympiques de Nagano: «Des loups, on en voit souvent dans la montagne. Peur? Non: ils filent toujours! (scappano sempre!)».

Nous avons voulu en savoir plus:

- Et si vous deviez vous perdre dans la montagne et passer la nuit sous un arbre ...?

- Ah, ça, on n'aimerait pas beaucoup ...

- Pourquoi donc?

- On ne sais pas, il pourrait y avoir une bête ...

- Quelle bête par exemple?

- Peut-être un sanglier ... (Magari un cinghiale ...) 


\section{Figure 3}

Le berger accompagné de ses chiens de la race des Abruzzes.

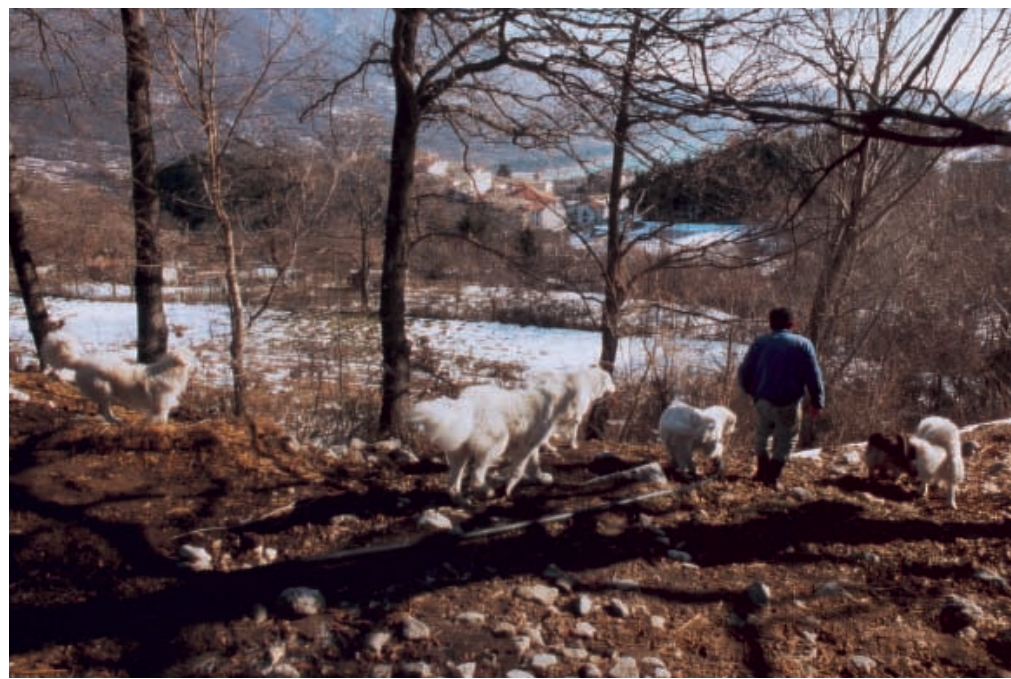

Impression confirmée par une jeune femme du village voisin: "Je suis du même avis: le sanglier était rare jusqu'à récemment et nous n'y sommes pas encore bien habitués, on se méfie ... tandis que le loup, on le connaît!»

Le même genre de témoignage vient d'autres régions d'Europe; de Pologne par exemple, où le ministre de l'environnement déclarait récemment à un journaliste suédois qu'aucun Polonais n'avait été attaqué par le loup dans ce pays qui en compte six cents (Dagens Nyheter du 16.12.2000). Jadis il est vrai, dans les régions rurales d'Europe, de nombreuses disparitions de jeunes enfants ont été attribuées au loup. En l'absence de contraception fiable, il naissait plus d'enfants que l'on pouvait en nourrir, et la pratique de l'infanticide semble avoir souvent été déguisée aux dépend du loup. Dès lors, la cérémonie funèbre pouvait avoir lieu, tout était bien et, une fois de plus, le loup avait le vilain rôle. Plus près de nous, la «bête du Gévaudan" était en fait un sadique, qui s'attaquait préférentiellement aux petites filles. Qui a dit que le l'homme est un loup pour l'homme? Ceux qui vivent avec les loups n'en ont pas plus peur que nous des renards.

\section{Le loup anéantit-il la faune sauvage?}

Nos premiers affûts étaient désespérants: des soirées et des aubes en toute discrétion aux meilleurs endroits signalés pas le garde, et: ni ours, ni loup. En revanche, quelle surprise de compter, à ce même endroit, jusqu'à trente-cinq cerfs dans notre champ de vision: des mâles aux bois superbes, des biches, une importante proportion de jeunes d'une ou deux années témoignant de la vitalité de la population, estimée à sept à huit cents individus actuellement. Nous voyions aussi des chevreuils, quoique moins souvent, les biotopes où nous observions en général leur convenant sans doute un peu moins bien. Une pensée en arrière: ces deux espèces ont été réintroduites il y a quelques décennies après avoir été exterminées par la chasse illégale. Leur réintroduction a été réalisée à partir d'un très petit nombre d'individus. Or, même à ce moment critique, cerfs et chevreuils n'ont pas été exterminés par le loup, et leurs effectifs ont pu croître régulièrement depuis lors. Nous avons aussi admiré une splendide troupe de chamois (118 adultes et 32 cabris en octobre 1989, excellente vitalité là également) de la sous-espèce des Apennins (ornata) qui avait failli être exterminée par la chasse: il n'en restait plus qu'une vingtaine dans les années soixante. Le développement de cette population a été tellement fructueux que l'on a pu en prélever des individus pour établir deux autres colonies dans les parcs nationaux du Gran Sasso d'Italia et de la Maiella, également dans la chaîne des Apennins. Les sangliers enfin sont fréquents dans le parc, trop peutêtre; taxés d'artefiziali parce qu'introduits et sélectionnés par les chasseurs des alentours, ils prolifèrent comme ... des sangliers et forment une part importante de la nourriture des loups.

Dans un biotope qui lui est plus favorable, en Toscane, le chevreuil cohabite aussi parfaitement avec le loup, ainsi que nous l'a signalé Luigi Boitani (déjà

\section{Figure 4}

Un cheval aux pattes antérieures entravées rencontré loin des villages: preuve que le loup ne constitue pas un danger.

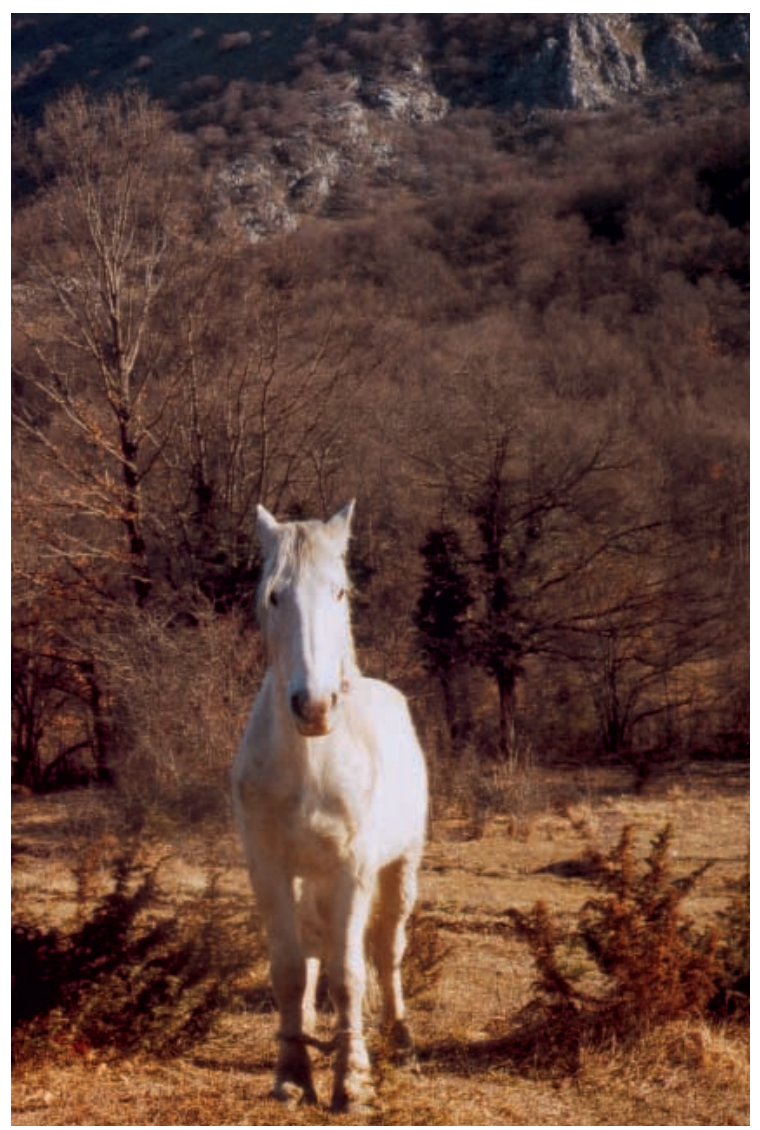


cité): en effet, non loin de Sienne, le loup est présent dans un secteur de 500 hectares peuplé de 25 chevreuils. En revanche, le chevreuil souffre beaucoup de la prédation des chiens errants (voir plus loin à ce sujet).

Hommes de terrain tout au long de l'année, les gardes nous ont confirmé que le loup et l'ours s'attaquent de préférence aux animaux affaiblis ou malades et contribuent ainsi, dans le sens de la sélection naturelle, à assurer l'excellente santé des espèces proies.

\section{Qu'en disent les éleveurs de moutons?}

Moins soutenu par l'Etat que chez nous (33 millions de subventions par an en Suisse!), l'élevage des ovins et caprins se pratique toujours dans le Parc et la production de fromage (pecorino, parfois fumé, affumicato) se fait de manière artisanale. A Civitella Alfedena, village hébergeant le Musée du Loup, nous avons découvert également une ... bergerie. Occasion d'aller à la rencontre de M. Corrado D'Andrea, éleveur, et de son berger croate.

Jusque dans les années septante, les pertes dues à l'ours et au loup étaient assez fréquentes en raison de la dégradation du milieu (moins d'arbustes à fruits pour l'ours; il en a été planté des milliers depuis lors) et surtout du manque de ressources alimentaires pour le loup: cerfs, chevreuils et autres proies potentielles avaient été exterminées par le braconnage avec la complicité corrompue et bienveillante de l'administration de l'époque. Le directeur actuel, Franco Tassi, est parvenu à mettre de l'ordre dans ce chaos, et notamment à réintroduire des cerfs (bavarois) et des chevreuils, désormais efficacement protégés contre le braconnage. Actuellement, malgré une augmentation substantielle du nombre des loups, les moutons sont en général épargnés: ainsi donc, tout le monde est

\section{Figure 5}

Maria Lucia Colantoni, propriétaire d'un restaurant à Villetta Barrea: «S'il n’y avait pas de loups et d'ours ici, je n'aurais pas de travail au village ..."

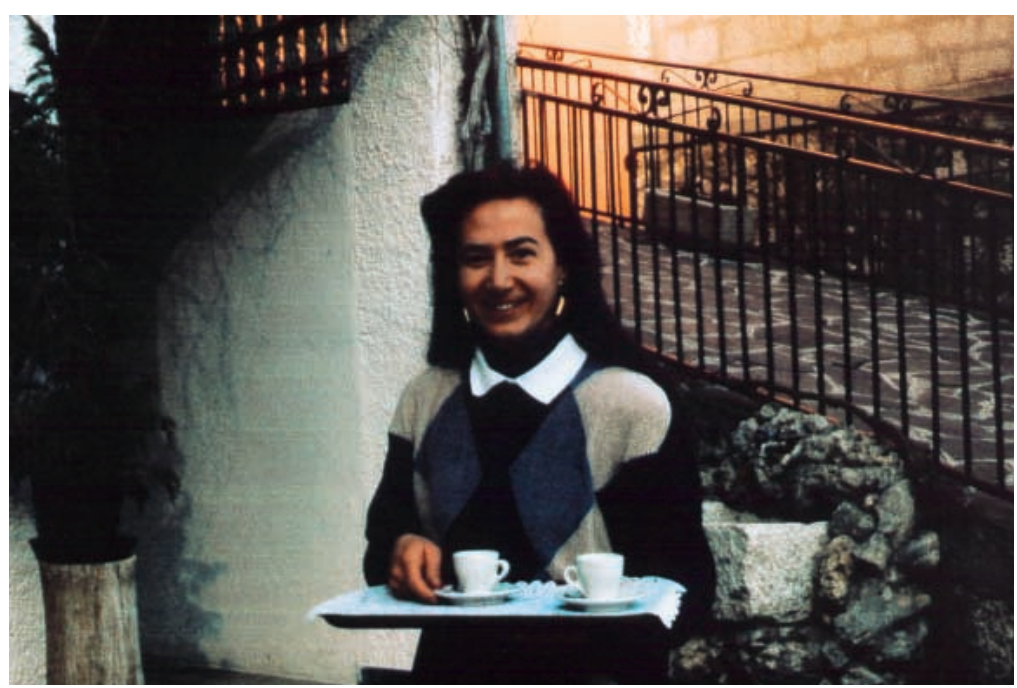

gagnant. Les cas d'attaque par des loups sont bien entendu indemnisés par l'administration du Parc (de l'ordre de 180000 lires pour une bonne brebis en 1999) même si dans bien des cas les fautifs sont sans doute des chiens errants (nous y reviendrons).

Après la traite, le berger emmène son troupeau vers les pâturages, accompagné de ses quatre chiens de la race locale. Le mastino abruzzese est un animal très pacifique, qui vient chercher des caresses auprès du promeneur et ne fait jamais de mal à un mouton (à la différence du patou des Pyrénées, essayé infructueusement au Val Ferret). Depuis sa naissance, il est élevé parmi les moutons, auxquels il va s'identifier, et le berger le renvoie d'un coup de pied s'il se rapproche trop souvent de lui; celui qui s'obstine à ne pas comprendre la leçon sera tué, manière dure mais efficace de sélectionner les individus les plus performants et de maintenir les qualités de la race. $\mathrm{Au}$ deuxième siècle avant Jésus Christ, l'auteur romain Columella décrivait déjà dans un traité d'agriculture un chien de berger en tout point comparable à l'actuel berger des Abruzzes. La sélection repose donc sur une très longue tradition.

De nuit, surtout par mauvais temps (tempo da lupo), que ce soit à l'étable ou, autrefois, dans les enclos en montagne, lorsque les chiens flairent l'approche des loups, ils se mettent à aboyer, comme pour signaler leur propre présence, en général pendant une heure environ, au bout de laquelle les loups se retirent (nous avons été témoins d'une telle scène une nuit de février 1999): sans doute savent-ils que, derrière un chien de berger, il y a un berger ... Il n'y a jamais de combats entre loups et chiens actuellement (mais on a dû les craindre autrefois si l'on en juge par les colliers à pointes exposés au Musée du Loup).

Jadis, nous a expliqué le vieux berger Nestore Campana, du temps de la transhumance, on comptait avec dix pour cent de pertes par an, en raison d'accidents, maladies, morsures de vipères, prédation par le loup ou l'ours. Pour compenser, on gardait chaque année trente-cinq agneaux dans un troupeau de trois cent cinquante bêtes. Aujourd'hui, avec en plus le problème relativement nouveau des chiens errants (voir ci-dessous), les pertes sont toujours du même ordre de grandeur, mais celles dues aux prédateurs sauvages sont indemnisées! Par ailleurs, les bergers d'autrefois ne se plaignaient pas toujours lorsqu'ils trouvaient un mouton tué: ils n'étaient de toutes façons pas personnellement propriétaires, et c'était parfois pour eux une occasion bienvenue de manger de la viande.

Plaie de l'Italie rurale, la présence depuis ces dernières décennies de centaines de milliers de chiens errants devenus plus ou moins sauvages (randagismo) est bien entendu redoutée des bergers, et aussi parfois des promeneurs: en effet, ces animaux sont parfois agressifs et de surcroît ils ne craignent pas l'homme. Il va sans dire qu'ils causent des dégâts considérables à la faune sauvage. Le loup est leur seul prédateur, quand il ne tombe pas sous le charme, ce qui donne parfois - heureusement très rarement naissance à des hybrides peu souhaitables. Les dé- 
charges publiques ayant été récemment interdites en Italie, on peut espérer que la disparition d'une offre de nourriture trop facilement accessible ralentisse au moins l'accroissement de cette population canine.

Rappelons ici que, en mars 1998, la presse signalait qu' "une horde de chiens sauvages a provoqué la mort de quelque 800 brebis affolées dans un pâturage de Catalogne (nord-est de l'Espagne). Prises de panique, les brebis ont péri, en majorité piétinées ou noyées dans des marécages ou dans un canal jouxtant la prairie où elles hivernaient». Plus récemment, "c'est un chien husky qui a dévasté un élevage de cerfs près de Beggingen ( $\mathrm{SH})$. Il a tué à la fin février 25 biches et 4 faons. Le dommage est évalué à quelque 30000 francs" (24 heures du 5.3.1999).

Dernièrement, des voix se sont élevées pour demander une réduction par le tir de la population des loups, jugée trop nombreuse par certains. Sensible à ces réactions émotionnelles, l'administration du Parc a alors lancé l'Operazione Arma Bianca, l'arme étant en l'occurrence le chien berger (blanc) dont on allait subventionner l'élevage et l'éducation pour pouvoir en offrir aux bergers, traités dès lors comme partenaires susceptibles de leur côté de fournir des observations sur les loups et les ours dans le terrain. Par ailleurs, de tels chiens seront également fournis à des bergers des Alpes, région où les chiens ont, au cours des générations, perdu l'habitude de réagir adéquatement à l'odeur du loup, qu'ils ne connaissent plus.

\section{Loups, chevaux et bovins}

A plusieurs reprises, notre attention a été attirée par des groupes de chevaux et de mulets paissant loin des villages, jour et nuit, même en hiver. Pour que leur liberté de mouvement ne soit pas trop grande, l'un des animaux du groupe est parfois entravé: ses deux pattes antérieures attachées l'une à l'autre l'empêcheraient bien entendu de fuir ou de ruer face à une attaque de la part des loups, ce qui prouve éloquemment que ce genre d'attaque ne se produit pas.

Il nous a été confirmé par ailleurs que les loups ne s'attaquaient pas non plus aux bovins.

Les observations faites ailleurs, au Canada notamment, de loups attaquant des élans ou des bisons ne sont pas transposables, essentiellement en raison de la taille nettement supérieure des loups de ces régions (jusqu’à soixante kilos).

\section{Le travail des gardes}

La surveillance du Parc est assurée par des gardes permanents, responsables chacun d'un secteur qu'il connaît bien. Les cas de braconnage sont rares. Notre ami Corrado Colantoni, garde à Villetta Barrea, se souvient d'un cas de braconnage de loup il y a quatre ans. En revanche, lorsqu'il a commencé son activité, on distribuait une prime pour chaque loup tué en Italie, même dans le Parc. Cette pratique a été abolie en 1972, le loup ayant été protégé par la loi du même coup.

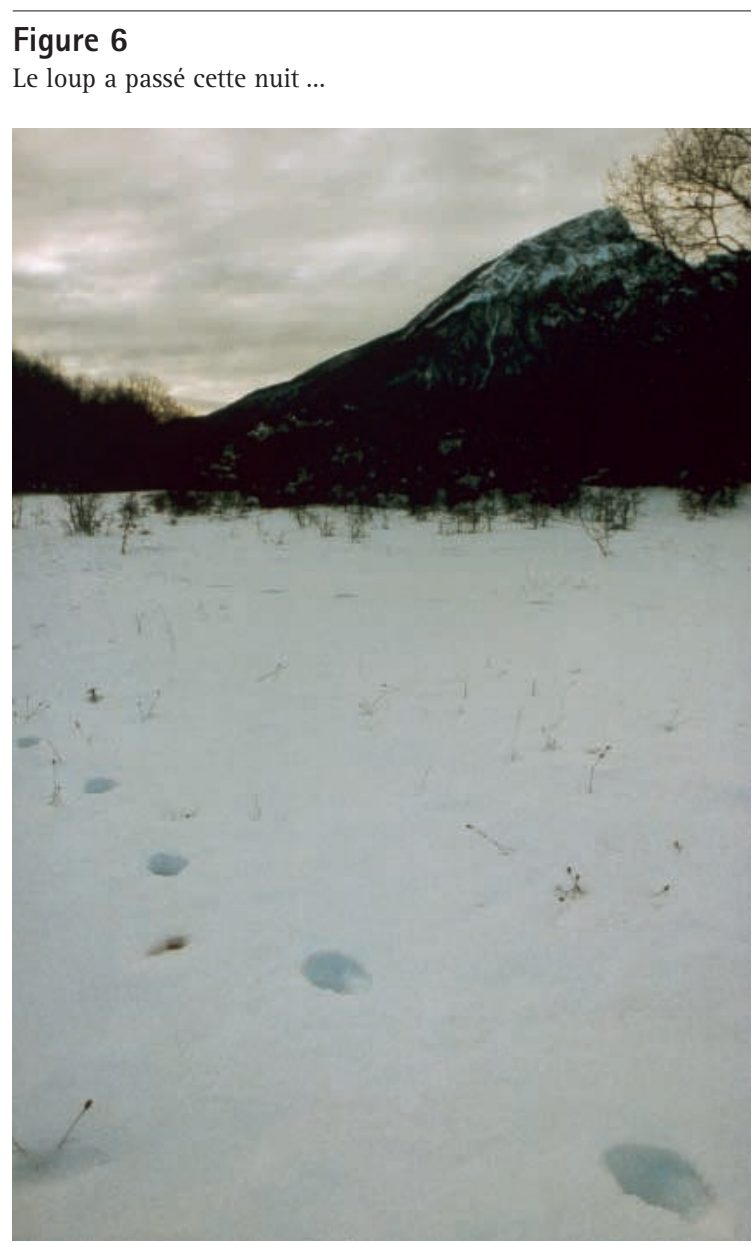

Une part importante de l'activité des gardes consiste en l'expertise des animaux domestiques signalés comme tués par les prédateurs sauvages. Il n'est pas rare que des propriétaires cherchent à abuser l'administration du Parc en revendiquant une indemnisation pour une bête morte d'une cause quelconque, puis offerte à des chiens qui lui infligent des morsures attribuées part la suite au loup. Un garde expérimenté ne s'y trompe guère et la fraude est dûment sanctionnée. En revanche, de jeunes gardes inexpérimentés ou manquant d'autorité se font parfois manipuler, d'où peut-être la forte augmentation des dépenses pour indemnisations constatée récemment. Un effort en vue d'un meilleur encadrement des jeunes gardes est en cours.

\section{En guise de conclusion}

Dans les vastes régions d'Europe d'où le loup a disparu, il est devenu habituel d'opposer les citadins, réputés assez favorables à son retour, aux campagnards, qui ne souhaiteraient pas sa réapparition. On oublie généralement d'interroger ceux qui ont le plus à nous apprendre: les personnes qui, depuis des générations, vivent avec les loups en bonne harmonie ou dans l'indifférence mutuelle. Il est essentiel d'al- 
Figure 7

Les enfants de Villetta Barrea: «Dès qu'il nous a vu, il a pris la fuite!»

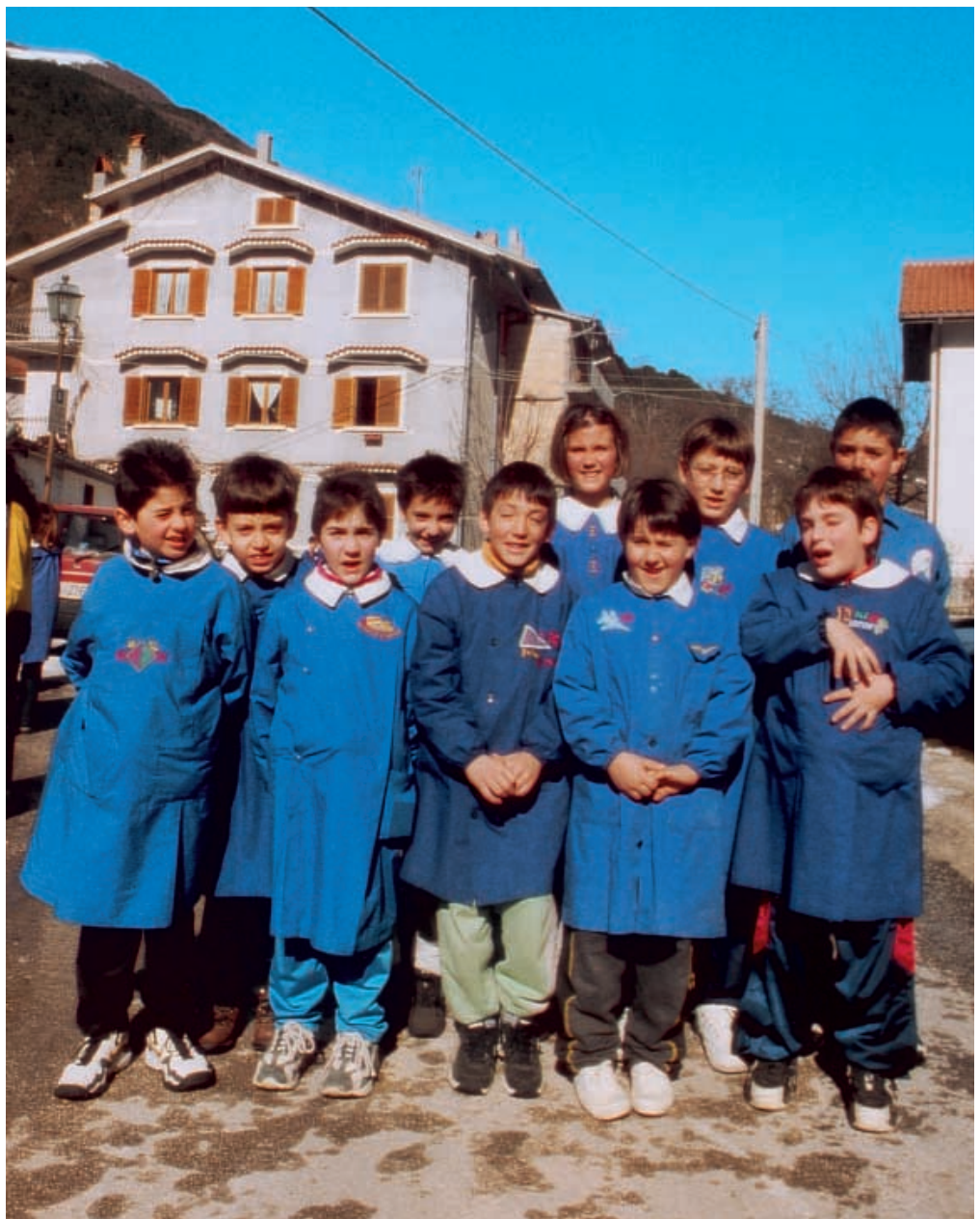

ler rencontrer ces gens, leurs témoignages valant plus que bien des théories, d'autant plus que les loups qui atteignent nos Alpes (les analyses de l'ADN l'ont prouvé) sont de souche italienne. C'est donc avec ces loups (et non pas avec ceux de Canada ou d'Europe de l'Est) que les comparaisons sont les plus valables.

Bien entendu, il ne s'agit pas ici de minimiser les problèmes que le loup peut causer à l'élevage des moutons tel qu'il est pratiqué actuellement en Suisse. En effet, contrairement à ce qui se passe dans les régions où le loup est présent, on a pris l'habitude chez nous de laisser les moutons à eux-mêmes la plupart du temps, souvent d'ailleurs par petits groupes qui ne justifieraient pas la présence continue d'un berger. La prévention des attaques et le dédommagement pour les bêtes blessées ou tuées nécessiteront des changements dans la gestion des troupeaux, et de nombreuses études sont en cours dans ce sens. On connaît déjà l'efficacité de certaines races de chiens ainsi que celle de bergers expérimentés (on a fait de meilleures expériences, dans les Abruzzes, avec des bergers ori- ginaires des Balkans, habitués au loup, qu'avec des Maghrébins). Par ailleurs, en Suède, on a observé que le loup est très méfiant à l'égard des clôtures: c'est ainsi que l'on a pu observer la trace dans la neige d'un loup qui arrivait droit sur une clôture de barbelés. Au lieu de la franchir, le loup l'a longée sur plusieurs kilomètres pour finalement la contourner. Tirant parti de ce genre d'enseignement, on a expérimenté des clôtures électriques, grâce auxquelles on a pu éviter toute prédation. En tout état de cause, ces mesures demanderont passablement d'efforts de la part de la collectivité, efforts qui - il faut le souligner - seront parfaitement vains si l'on ne parvient pas à contrôler efficacement les chiens "accompagnant" des promeneurs peu scrupuleux ou incapables de les maîtriser. Un berger valaisan me confiait d'ailleurs que ce problème le préoccupait bien plus que celui, infiniment plus médiatique, du loup.

Une certaine évolution s'observe par ailleurs chez les chasseurs, dont certains entrevoient que la faune sauvage constitue un ensemble cohérent et acceptent de ne plus considérer les prédateurs sauvages comme des ennemis mais comme des éléments essentiels de cet ensemble, au sein duquel ils jouent un rôle sanitaire que la chasse a de la peine à assurer. Saluons ici l'attitude clairvoyante et moderne du président de la Diana de Conches, en Haut-Valais, Helmut Kiechler, qui "s'élève contre le fait que des lynx sont abattus dans notre canton comme si c'était la chose la plus naturelle du monde.(...) Il proteste contre les battues sauvages à l'encontre du loup. Le Conseil d'Etat ferait mieux, selon lui, d'élaborer le dédommagement pour les dommages causés, plutôt que de verser de l'huile sur le feu». (le Nouvelliste du 24 mai 1996).

On l'a vu, le retour du loup va faire encore quelques remous, et c'est bien compréhensible. Mais une chose en tout cas est désormais certaine: toute personne qui prétend que l'arrivée en Suisse de loups sauvages peut constituer un danger pour la population humaine est soit ignorante, soit de mauvaise foi. On n'a plus le droit d'insinuer que des accidents pourraient survenir, comme nous l'avons encore entendu à deux reprises récemment en public de la part de personnes qui devraient être correctement instruites. Allez demander aux enfants des Abruzzes!

\section{Mes remerciements ...}

vont à toutes les personnes qui m'ont accueilli, m'ont aidé et ont répondu à mes questions au Parc National des Abruzzes depuis 1989, tout particulièrement à son directeur le Dr Franco Tassi et au garde Corrado Colantoni, ainsi qu'au Professeur Luigi Boitani de l'Université de Rome.

Les habitants de l'Alto Sangro m'ont appris que le loup est un animal comme les autres. Mais quand même pas tout à fait comme les autres ... Et surtout que, plus on l'étudie, plus il nous en apprend - aussi sur nous-mêmes. 